Notas técnicas 



\title{
CRÍTICA DEL CONCEPTO DE EXERGÍA
}

\author{
René Alejandro Mora Casal
}

\begin{abstract}
Resumen
La exergía es un concepto que, desde su invención hace sesenta años, ha ido ganando popularidad y extendiéndose más allá de las aplicaciones de Ingeniería. Sin embargo, un estudio a profundidad del concepto revela la existencia de problemas e inconsistencias, tanto de tipo teórico como de aplicación. En este documento se discuten seis problemas identificados: notación ambigua, estados de referencia ambiguos, aplicabilidad a procesos reales, redundancia con respecto a otras propiedades termodinámicas, inconsistencia entre los conceptos de exergía y de trabajo perdido, y la aplicabilidad de los resultados del análisis exergético. Se proponen posibles caminos de solución para estos problemas, siendo el quinto el de más difícil solución porque requiere redefinir qué es la exergía.
\end{abstract}

Palabras clave: Fuerza y energía; termodinámica-modelos matemáticos; análisis térmico; termodinámicamedición; física.

\begin{abstract}
Exergy is a concept that, since its invention sixty years ago, has gained popularity and has extended beyond engineering applications. However, a deep study of this concept reveals problems and inconsistencies, both of theoretical type and of application. In this article six problems are identified and discussed: ambiguous notation, ambiguous reference states, applicability to real processes, redundancy with respect to other thermodynamic properties, inconsistency between the concepts of exergy and lost work, and applicability of the exergetic analysis results. Some roads to solution for these problems are proposed, being the fifth one the most difficult to solve, as it requires a redefinition of exergy.
\end{abstract}

Keywords: Strength and energy; thermodynamics-mathematical models; lost work; second law, thermal analysis.

Recibido: 18 de Junio 2014 Aprobado: 13 de Octubre 2014

\section{INTRODUCCIÓN}

La exergía es un concepto que se definió por primera vez hace sesenta años, el cual ha adquirido gran relevancia en la actualidad, pues encuentra cada día nuevas aplicaciones. Inicialmente se utilizó para la optimización de procesos energéticos, pero en los últimos años ha salido del ámbito industrial y está siendo utilizado por profesionales en otros campos como la Ecología, la Biología y la Arquitectura (ej. Ayres, Ayres, Martinas, 1996; Simpson, Edwards,
2011; Meggers, Ritter, Goffin, Baetschmann, Leibundgut, 2012).

A pesar de lo anterior, el concepto de exergía presenta algunos problemas a nivel teórico y de aplicación. El propósito del presente ensayo es hacer un análisis crítico de dicho concepto, presentando los diferentes problemas que este autor ha identificado y analizándolos con el fin de proponer posibles soluciones. No forma parte del alcance de este documento una enumeración y análisis exhaustivos de todos los problemas o limitaciones asociados con el análisis exergético; 
al respecto otros autores han analizado este tema desde otras perspectivas: Linnhof, 1979; Tsatsaronis, 1999; Zvolinsky, 2006; Tsatsaronis, 2007; Morosuk \& Tsatsaronis, 2009). Tampoco se pretende comentar sobre los aspectos positivos del uso de la exergía en el análisis de procesos, o sobre los algoritmos matemáticos e informáticos que se han desarrollado en años recientes, dado que ya existe mucha literatura que trata estos temas, por el contrario los aspectos de crítica se han documentado muy poco.

La primer parte definirá la exergía y se comentará sobre la evolución que este concepto ha tenido en los últimos 40 años. En una segunda parte se analizarán los problemas encontrados con respecto a este concepto y que de alguna manera dificultan su respaldo teórico o su aplicación directa. Finalmente, se propondrán posibles alternativas para resolver los problemas planteados.

\section{CONCEPTO DE EXERGÍA Y SU APLICACIÓN}

La exergía es una propiedad de los sistemas termodinámicos que permite incluir el entorno en los cálculos; se puede considerar como una medida de la "calidad" de la energía disponible y surge del análisis de un proceso con base en las dos primeras leyes de la Termodinámica. Una de sus posibles definiciones es:

La cantidad de trabajo obtenible cuando alguna materia se lleva a un estado de equilibrio termodinámico con los componentes comunes del entorno natural por medio de procesos reversibles. (Szargut, Morris, Steward, 1988).

Otra definición afirma que "es trabajo o la habilidad de producir trabajo" (Dincer \& Cengel, 2001). El estado final se conoce como estado muerto (Rosen \& Dincer, 2004).Como se verá más adelante, el concepto obtenible (u otro término similar) en la definición de exergía es cuestionable.

A diferencia de la energía que se conserva, la exergía puede ser destruida durante un proceso irreversible; sin embargo, la exergía absoluta solo puede ser positiva o cero, por definición (Dincer \& Cengel, 2001). En términos matemáticos, la exergía se puede definir mediante cualquiera de las siguientes ecuaciones, las cuales se puede demostrar que son equivalentes (Wall, 1977). La Tabla 1 muestra los significados de las siquientes variables. (Ver ecuaciones 1, 2 y 3)(Ver Tabla 1)

$$
\begin{aligned}
& E=U+p_{0} V-T_{0} S-\sum \mu_{i 0} n \\
& E=S\left(T-T_{0}\right)-V\left(p-p_{0}\right)+\sum n_{i}\left(\mu_{i}-\mu_{i 0}\right)
\end{aligned}
$$

La definición anterior es válida dentro de un volumen de control; para las corrientes que entran y salen del sistema, $U+p \_O V$ se debe sustituir por H. Según la necesidad, a las Ecuaciones (1), (2) y (3) se les pueden añadir otros términos tales como el de energía cinética, energía potencial y difusión química (Wall, 1977; Abata, 2011).

Como un breve ejemplo, considérese una turbina de vapor con una potencia nominal de $56400 \mathrm{~kW}$. Vapor sobrecalentado entra a 8600 $\mathrm{kPa}$ y $500^{\circ} \mathrm{C}$, y se descarga saturado a una presión de $10 \mathrm{kPa}$. La pérdida de calor es despreciable y la eficiencia isentrópica de la turbina es 0,75 . Se supondrá que la temperatura del estado muerto es $25^{\circ} \mathrm{C}$. Con base en esta información se pueden calcular los valores de $\mathrm{H}$ y $\mathrm{S}$, para después calcular las exergías de entrada y salida de esta manera:

$\mathrm{E}_{1}=\mathrm{H}_{1}-\mathrm{T}_{0} \mathrm{~S}_{1}=3391,6-298.15 * 6,6858=1398.23 \mathrm{~kJ} / \mathrm{kg}$

$\mathrm{E}_{1}=\mathrm{H}_{1}-\mathrm{T}_{0} \mathrm{~S}_{1}=2435,95-298.15 * 7,6846=144.79 \mathrm{~kJ} / \mathrm{kg}$

Estos datos servirán para hacer otros cálculos posteriormente.

El término exergía fue acunado por Z. Rant en 1953 y literalmente significa "capaz de un trabajo externo" (Sciubba \& Wall, 2007). Antes y después de esa fecha, se han usado distintos términos equivalentes a este concepto: capacidad técnica de trabajo, disponibilidad, energía disponible, trabajo disponible, trabajo potencial, energía útil, esergía o energía esencial, entropía potencial. Los primeros escritos sobre el concepto de energía disponible se atribuyen a J. W. Gibbs en 1873 (Ayres et al, 1996; Sciubba \& Wall, 2007).

$$
E=U-U_{e q}+\mathrm{p}_{0}\left(V-V_{e q}\right)-T_{0}\left(S-S_{e q}\right)-\sum \mu_{i 0}\left(n_{i}-n_{i, e q}\right)
$$


Tabla 1. Variables incluidas en Ecuaciones 1-3

\begin{tabular}{lcc}
\hline \multicolumn{1}{c}{ Variable } & Sistema & Alrededores= Estado muerto \\
\hline Energía & $\mathrm{E}$ & Igual a cero \\
Energía Interna & $\mathrm{U}$ & $\mathrm{U}_{\mathrm{eq}}$ \\
Presión & $\mathrm{p}$ & $\mathrm{p}_{0}$ \\
Volumen & $\mathrm{V}$ & $\mathrm{V}_{\mathrm{eq}}$ \\
Temperatura & $\mathrm{T}$ & $\mathrm{T}_{0}$ \\
Entropía & $\mathrm{S}$ & $\mathrm{S}_{\mathrm{eq}}$ \\
Potencial químico & $\mu_{\mathrm{i}}$ & $\mu_{\mathrm{i} 0}$ \\
Cantidad de substancia & $\mathrm{n}_{\mathrm{i}}$ & $\mathrm{n}_{\mathrm{i}, \mathrm{eq}}$ \\
\hline
\end{tabular}

Nota: Adaptado de "Exergy - A Useful Concept", por G. Wall, 1977, Chalmers Institute of Technology: Göteborg, Sweden.

Antes de 1950 era casi inexistente la literatura sobre exergía (Wall, 1992). Así por ejemplo, el libro Textbook of Physical Chemistry de Glasstone (1946) no hace mención del término. Quince años después, la primera edición del libro Fundamentals of Classical Thermodynamics de Van Wylen y Sonntag (1965) dedica un capítulo al tema "Irreversibilidad y disponibilidad", siendo este último término un sinónimo de la exergía.

Actualmente, casi todos los libros de Termodinámica incluyen un capítulo sobre exergía o balance exergético; una excepción notable es el libro An Introduction to Chemical Engineering Thermodynamics de Smith, Van Ness y Abbott, que en su sétima y última edición (2004) no incluye este concepto. Tampoco se menciona la exergía en la octava y última edición del Perry's Chemical Engineers' Handbook (2008). En la Figura 1 se muestra el número de publicaciones sobre exergía que han aparecido en forma anual entre los años 1940 y 1992.
El análisis exergético ha sido aplicado principalmente en los procesos de generación de potencia (turbinas y motores), propios de la ingeniería mecánica, dado que la existencia de tablas de propiedades termodinámicas para diferentes fluidos facilitan el cálculo de los cambios de exergía (Van Wylen \& Sonntag, 1976; Wall, 1986). Sin embargo, la exergía también se ha aplicado en el campo de la ingeniería química; por ejemplo: el análisis de procesos industriales, el diseño de procesos de destilación y desalinización y el diseño de redes de intercambiadores de calor (Linnhof, 1979; Kaiser \& Gourtia, 1985; Zhihong \& Ben, 2000; Faria \& Zemp, 2005; Sciubba \& Wall, 2007). Actualmente, la aplicación del concepto de la exergía se está extendiendo a otros campos, como la ecología industrial y el diseño arquitectónico (Connelly, Koshland, 2001; LowEx, 2002; Shukuya, 2005; Zvolinsky, 2006; Meggers, 2012).

Un campo relacionado con la exergía es el de la Termoeconomía, disciplina creada en el 


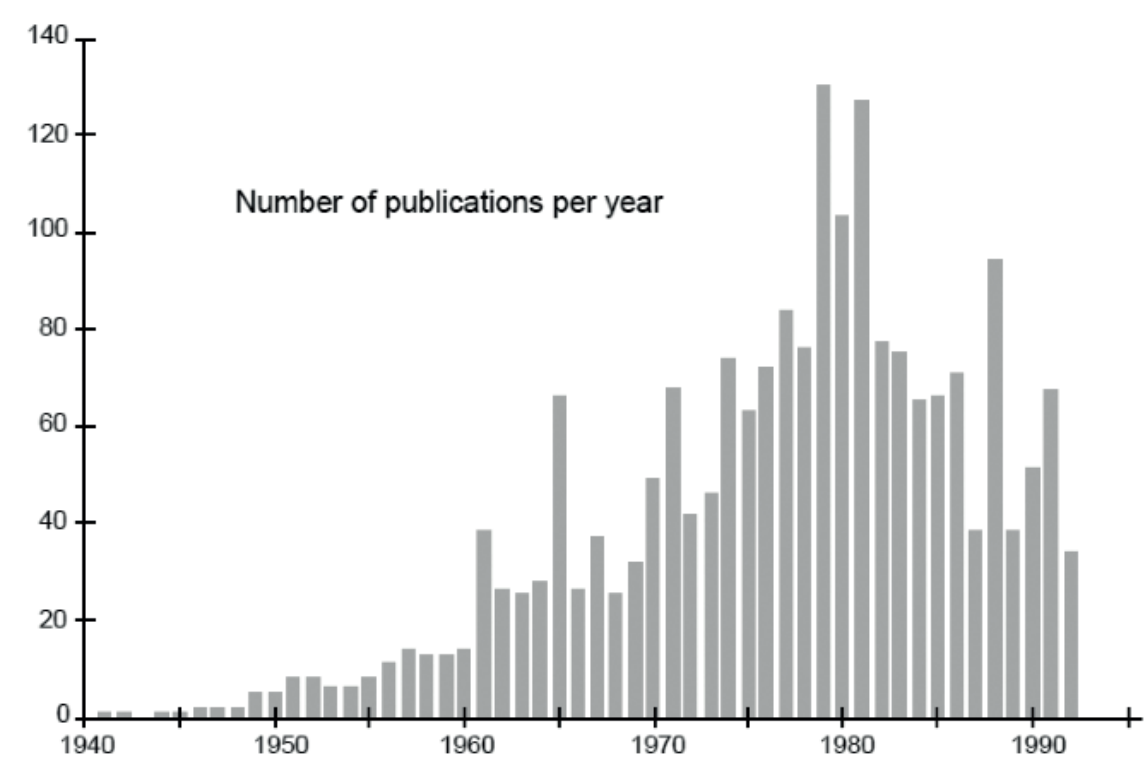

Figura 1. Artículos sobre exergía publicados entre 1940 y 1992.

Nota: Tomado de “A Bibliography of Exergy”, por G. Wall, 1992.

Massachussets Institute of Technology en 1961 y que se refiere a la combinación del análisis exergético y la ingeniería económica: consiste en asignar un costo asociado a las pérdidas causadas por la irreversibilidad en los procesos, lo que permite incluir el criterio termodinámico en los estudios de factibilidad económica y la toma de decisiones sobre proyectos. Esta disciplina ha tenido auge en años recientes gracias al desarrollo de nuevos y poderosos algoritmos matemáticos (Sciubba \& Wall, 2007).

\section{PROBLEMAS CON EL CONCEPTO DE EXERGÍA}

El concepto de exergía tiene algunos problemas reconocidos por los expertos en el tema (Linnhof, 1979; Tsatsaronis, 1979); varios de estos problemas permanecen abiertos y son objeto de discusión en la actualidad (Ayres et al, 1996; Zvolinsky, 2006; Sciubba \& Wall, 2007). Seguidamente se procederá a describir cada uno de los problemas identificados en el ámbito de este trabajo.

\subsection{Notación ambigua}

Uno de los primeros problemas que se encuentran al iniciar el estudio de la exergía es el hecho de que no hay una notación estándar, de tal manera que diferentes autores usan símbolos distintos para esta propiedad (Sciubba \& Wall, 2007). Algunos de los símbolos usados son A, B, E, Ex, e, X, $\phi$. La desventaja de esto es que se dificulta la comunicación entre profesionales por el uso de diferentes símbolos en las fórmulas y ecuaciones, a la vez que puede haber una confusión con otras propiedades termodinámicas, por ejemplo: la energía de Helmholtz (A), el segundo coeficiente virial (B) o el coeficiente de fugacidad $(\phi)$, entre otros.

\subsection{Estados de referencia ambiguos.}

Otro de los problemas de la exergía es la estandarización del estado de referencia o estado muerto, importante para la unificación de la disciplina (Ayres et al, 1996). Con esto se hace referencia a que la definición del estado 
muerto implica la necesidad de establecer una presión, una temperatura y una composición de referencia que, preferiblemente, sean las mismas para todo el mundo con el fin de que el análisis de un proceso no dependa de su ubicación. La presión de referencia es fácil de definir; la temperatura no lo es tanto, y la composición es francamente difícil, por razones de variabilidad (Martínez, 1992). Se han podido definir exergías de referencia para elementos y compuestos, con base en las energías de Gibbs de formación y de las entalpías de combustión: lo anterior es bastante directo en el caso de productos de combustión, pero no lo es tanto en el caso de compuestos metálicos, halogenados y de otros tipos; esto determina que existan varias propuestas de tablas para las exergías de referencia de elementos y compuestos con distintos valores (Ayres et al, 1996).

Muy relacionada con el tema anterior se encuentra la necesidad de establecer una atmósfera, una hidrosfera (océano) y una litosfera (corteza terrestre) de referencia, dependiendo de si los productos finales del proceso son gaseosos, líquidos o sólidos. La atmósfera de referencia es relativamente fácil de definir, mientras que la hidrosfera y la litosfera plantean importantes dificultades, también por razones de su gran variabilidad, a pesar de lo cual muchos investigadores han hecho propuestas en este sentido. Una consecuencia es que los valores de exergía pueden diferir sustancialmente, dependiendo de la definición del estado de referencia (Ayres et al, 1996).

\subsection{Aplicabilidad a procesos reales}

Un tercer problema que presenta el concepto de exergía está relacionado con una de las limitaciones de la termodinámica en sí, específicamente el concepto de reversibilidad. Con esto se hace referencia a que el análisis exergético, como todo análisis basado en las leyes de la Termodinámica, parte de la existencia de uno o más procesos reversibles -y por tanto ideales- para poder calcular el trabajo máximo (o mínimo) que puede entregar el sistema, asumiendo, por ejemplo, eficiencias de Carnot y reemplazando $Q$ por $T d S$. Para calcular el trabajo perdido o la exergía destruida se requiere hacer mediciones del desempeño o las eficiencias reales; por tanto, con el concepto de exergía no se elimina esta necesidad. Más adelante se analizará una inconsistencia en la relación entre la exergía y el trabajo perdido.

Se ha intentado aplicar la teoría termodinámica de los procesos irreversibles a la exergía (Wall, 1977). Un progreso sustancial en esta teoría resulta necesario para poder avanzar en la correcta aplicación de la exergía a los procesos biológicos y ecológicos, aplicación que según algunos autores todavía está poco fundamentada, pese a que en años recientes se han se han realizado investigaciones y avances al respecto (Zvolinsky, 2006; Sciubba \& Wall, 2007; Simpson \& Edwards, 2011).

\subsection{Redundancia con respecto a otras propiedades}

Otro problema de tipo teórico o conceptual es la aparente redundancia del concepto de exergía con respecto a otros conceptos estrechamente relacionados con ella, como la entropía, la energía libre y el trabajo perdido: surge la pregunta si realmente se requería un concepto nuevo. Varios autores señalan que la exergía (E) es una especie de propiedad "comodín", la cual puede adoptar la identidad de otras propiedades tales como la energía interna (U), la entalpía (H), la energía de Helmholtz (A), o la energía de Gibbs (G), dependiendo de las condiciones del sistema (e.g. Wall, 1977). Se profundizará un poco con respecto a este punto particular.

La definición de exergía (Ecuaciones 1 3 ) involucra una expresión de la forma $U+$ $P V-T S$, que podemos expresar de la siguiente manera, en forma diferencial exacta:

$d E=\left(d U-T_{0} d S\right)-\left(-p_{0} d V\right)$

El primer término entre paréntesis es similar a la energía de Helmholtz (dA), la cual también es conocida como función de trabajo, ya que representa el máximo trabajo $W$ que se puede obtener del sistema a través un proceso isotérmico reversible, como se demuestra a continuación (Glasstone, 1946): 
$d U=Q+W$

(Primera Ley)

$Q=T d S$

(Segunda Ley)

$d A=d U-T d S$

(definición de dA)

$d A=W$

El segundo término en la Ecuación 4 es similar al trabajo de expansión $W_{\text {exp }}$ que se realiza desde el sistema, o sobre el sistema si es de compresión. El signo negativo dentro del paréntesis toma en cuenta que $W$ y $d V$ tienen por definición signos contrarios (p. ej. durante una compresión del sistema, $d V$ es negativo pero $W$ es positivo). Por tanto, el miembro derecho de la Ecuación 4 corresponde al máximo trabajo que se puede obtener del sistema que es distinto del trabajo de expansión, y se puede demostrar que este trabajo es igual al cambio en la energía de Gibbs (Glasstone, 1946):

$d G=d U-T d S-(-p d V)$ a $P, T$ constantes (definición de $d G)$

$d G=W-W_{\text {exp }}$

$d G=W_{\text {no } \exp }$

La línea de razonamiento anterior tiene como propósito mostrar la estrecha relación entre la exergía y la energía de Gibbs, que coinciden cuando $p=p_{0}$ y $T=T_{0}$, y que todos los análisis exergéticos son en el fondo análisis basados en esta última propiedad. El único argumento en contra sería que la definición de exergía utiliza la presión y temperatura de los alrededores, mientras que la definición de la energía de Gibbs utiliza la presión y la temperatura del sistema. Aun así, existe una relación entre ambas propiedades, como se ve a partir de las definiciones de $d E$ y $d G$ anteriores, de donde se deduce lo siguiente:

$d E=d G+\left(p_{0}-p\right) d V-\left(T_{0}-T\right) d S$

Una corroboración de los argumentos anteriores yace en el hecho de que las exergías de los materiales se suelen calcular a partir de las energías de Gibbs de formación (Ayres et al, 1996; Simpson \& Edwards, 2011). Adicional a lo anterior, la primera definición de energía disponible de la que se tiene noticia, la cual se cita como una de las primeras referencias al concepto de exergía (ej. Wall, 1977; Sciubba \& Wall, 2007), corresponde en realidad a la definición de energía de Gibbs:
...the greatest amount of mechanical work which can be obtained from a given quantity of a certain substance in a given initial state, without increasing its total volume or allowing heat to pass to or from external bodies, except such as at the close of the processes are left in their initial condition. (Gibbs, 1873).

\subsection{Inconsistencia entre exergía y trabajo perdido}

Un problema adicional se presenta cuando se trata de relacionar la exergía con el concepto de trabajo perdido o irreversibilidad, ya que se presenta una inconsistencia, lo cual se demuestra a continuación, por lo menos a nivel de la definición. El trabajo perdido durante un proceso se puede definir mediante las siguientes fórmulas (Smith et al, 2001):

$W_{\text {perd }}=W_{\text {real }}-W_{\text {ideal }}$

$W_{\text {perd }}=T_{0} \Delta S_{\text {total }}$

Las ecuaciones anteriores son rigurosas y aplicables tanto en procesos donde el trabajo entra al sistema (ej. compresión, máquina frigorífica) como en aquéllos donde el trabajo sale del sistema (ej. expansión, máquina térmica): en el primer caso los dos términos $W_{\text {real }}, W_{\text {ideal }}$ son positivos y $W_{\text {real }}>W_{\text {ideal }}$, mientras que en el segundo caso los dos términos W_real, W_ideal son negativos y $\left|W_{\text {real }}\right|<\left|W_{\text {ideal }}\right|$. Por tanto, en ambos casos se cumple que el trabajo perdido es siempre positivo.

Por otra parte, diversos autores indican que la exergía absoluta no puede ser negativa (ej. Wall, 1977; Abata, 2011) y deducen la siguiente relación entre la exergía, el trabajo real y la entropía total (Wall, 1977):

$W=E-T_{0} \Delta S_{\text {total }}$

Esta ecuación es correcta si (i) se adopta la convención de que el trabajo es positivo cuando sale del sistema (seguida por G. Wall y otros autores), 
contrario a la más moderna y utilizada aquí, y (ii) todas las variables son positivas, correspondiendo a un caso de expansión o máquina térmica y asimismo a la definición de exergía. Sin embargo, en el caso de compresión o máquina frigorífica la ecuación es inconsistente porque la exergía $E$ tendría que ser negativa. Esta inconsistencia se resuelve al concluir que en realidad $E$ es una diferencia de exergía $\Delta E$, pudiendo ser entonces negativa. Esto no es claro en la fuente original.

Si se adopta la convención de que el trabajo es positivo cuando entra del sistema, la Ecuación (10) toma la siguiente forma que es consistente con la Ecuación (8):

$W=\Delta E+T_{0} \Delta S_{\text {total }}$

Retomando el ejemplo introducido en la segunda sección, y con base en el caudal de vapor de $59,02 \mathrm{~kg} / \mathrm{s}$, se puede corroborar lo siguiente:

$W_{\text {real }}=W=\dot{m} \Delta h=-56400 \mathrm{~kW}$

$W_{\text {ideal }}=\Delta E=\dot{m} \Delta e=-73974,9 \mathrm{~kW}$

$W_{\text {perd }}=T_{0} \Delta S_{\text {total }}=\dot{m} T_{0} \Delta s=+17574,9 \mathrm{~kW}$

Con respecto a la definición usual de la exergía, se observa que ésta es deficiente o inconsistente en el sentido que hace referencia a "trabajo obtenible" (Szargut et al., 1988) o "trabajo máximo teórico que se puede extraer del sistema" (Sciubba \& Wall, 2007), mientras que en la realidad hay procesos en los que el trabajo teórico o ideal es añadido al sistema y será mínimo. Por tanto esta definición no tiene la generalidad que muestran las Ecuaciones (8) y (11).

\subsection{Aplicabilidad de los resultados del análisis exergético}

Un problema final con el concepto de exergía radica en el hecho que sus resultados y recomendaciones no siempre son aplicables o realizables. Dicho en forma más precisa, un balance de exergía no es suficiente para valorar el beneficio de una propuesta de mejora de un proceso, sino que es necesario hacer una comparación de los balances energéticos globales del proceso original y del proceso mejorado, y además constatar la factibilidad energética y económica de la nueva alternativa (Pisa, Agüero, Andina, 2010). Estos autores presentan ejemplos en donde el análisis exergético no aporta una mejora en el aprovechamiento energético y económico, por ejemplo al requerir fuentes de energía costosas (ej. una caldera) en vez de aprovechar recursos energéticos de bajo costo (ej. recuperación de calor).

Otros autores han señalado otras limitaciones en el análisis exergético (Linnhof, 1979; Tsatsaronis, 1999, 2007; Zvolinsky, 2006; Morosuk \& Tsatsaronis, 2009), que no se tratarán aquí.

\section{DISCUSIÓN Y CONCLUSIONES}

El análisis exergético de un sistema genera los mismos resultados que un análisis convencional basado en la Segunda Ley de la Termodinámica, $\mathrm{y}$ de alguna manera ha permitido que las consideraciones termodinámicas empiecen a tomarse en cuenta en el diseño de procesos industriales y en los análisis de tipo ecológico y urbanístico. El auge que ha venido tomando el concepto en las últimas décadas se debe a varias posibles razones: reúne un conjunto de términos que aparecen repetidamente en el análisis termodinámico de sistemas; se asocia fácilmente con la energía y permite hacer comparaciones; permite "penalizar" las etapas ineficientes de un proceso durante el análisis con el fin de identificar oportunidades de mejora; organizaciones importantes como el Massachusetts Institute of Technology se han interesado en desarrollar y aplicar el concepto; cada vez hay más libros y publicaciones sobre el tema; y hay un número creciente de profesionales y expertos que aplican y promueven este tipo de análisis.

En relación con el problema de la notación, se puede considerar de fácil solución, y resulta sorprendente que todavía no haya surgido una iniciativa para uniformarla, pues esto contribuiría a aumentar la claridad de los conceptos, las fórmulas y las ecuaciones, tanto a nivel de la enseñanza como de la divulgación. Esto requeriría de uno o más congresos internacionales donde se discuta este tema y se llegue a un consenso.

El problema del estado muerto o de referencia es más difícil, pero no imposible de 
resolver y de hecho es un área activa de trabajo de investigación en donde ya se han alcanzado avances importantes (Szargut et al, 1988; Ayres et al, 1996; Rosen \& Dincer, 2004; Zvolinsky, 2006; Pons, 2009). Es opinión de este autor que eventualmente se llegará a un consenso en cuanto a la definición del estado de referencia y al manejo de las variaciones locales y estacionales.

Es problema de la reversibilidad, no es solamente una limitación del análisis exergético, sino del análisis termodinámico en general, y por tanto es de difícil solución. Existe una termodinámica de procesos irreversibles que se ha tratado de aplicar al análisis exergético; sin embargo, persiste la limitación de que los procesos deben cumplir el principio del equilibrio local, lo cual no se cumple en la mayoría de los procesos industriales, biológicos y ecológicos (Wall, 1977; Sciubba \& Wall, 2007). Esta área constituye un campo de investigación activo y en el que se han dado avances recientes (ej. Zvolinsky, 2006). Para el cálculo de las irreversibilidades se seguirá dependiendo de la información acumulada sobre eficiencias de equipos y procesos, coeficientes fenomenológicos y otros datos, así como de la experiencia y el buen juicio del profesional.

La redundancia del concepto de la exergía no es necesariamente un problema, ya que a nivel de la Termodinámica se han definido muchos conceptos y variables, debido a necesidades que no eran cubiertas por los conceptos anteriores. La justificación para definir y utilizar el concepto de exergía podría considerarse de orden práctico. Por la misma razón, resulta optativo para el profesional utilizar el análisis exergético o bien el análisis termodinámico convencional, y así sucede en la práctica en donde se combinan ambos.

El problema de la inconsistencia al tratar de relacionar la exergía con el trabajo perdido -a través de la Ecuación 10- resulta ser principalmente un problema de ambigüedad de la notación, al escribir unos autores $E$ en lugar de $\Delta E$, por lo que este punto se puede considerar resuelto en este trabajo. En cambio, para resolver la inconsistencia que presenta la definición de exergía, se requiere revisar el desarrollo teórico y conceptual del análisis exergético, con el fin de que dicha definición sea totalmente rigurosa y general, que cumpla con la Ecuación 8 y que no se limite a hacer referencia a un trabajo máximo o trabajo obtenible.

Finalmente, el problema de la aplicabilidad de los resultados del análisis exergético está relacionado con la práctica profesional, en el sentido que los errores y los malos resultados se pueden dar cuando se realiza un análisis superficial del proceso (Pisa et al, 2010). Por tanto, la experiencia y el criterio del profesional serán siempre de suma importancia a la hora de definir la herramienta conceptual a utilizar. Un autor importante ha argumentado que una de las supuestas debilidades del análisis exergético, a saber la falta de una metodología estructurada y su carácter completamente general, constituye a la vez una de sus mayores fortalezas ya que no le impone restricciones a la imaginación y a la creatividad del ingeniero (Tsatsaronis, 1999).

Se finaliza este ensayo con un pensamiento del experto en exergía Goran Wall, que consideramos muy apropiado en relación con los temas aquí tratados:

It must be noted that thermoeconomics can never replace long experience and high technical and economic competence. Manufacturers will always be driven by two strong driving concerns: minimum cost and minimum performance standards. However, if they considered the system as a way to produce heat they should offer minimum cost of heat rather than minimum cost of poor equipment. In any case, thermoeconomics will always be an important complementary tool. (Wall, 1991).

\section{NOTAS}

1. Ejemplo 7.6 del texto de Termodinámica de Smith, Abbott y Van Ness, sexta edición (2002).

\section{REFERENCIAS BIBLIOGRÁFICAS}

Abata, D. (2011). The Concept of Exergy. Notes for the courses ME211 Thermodynamics $I$ and ME 312 Thermodynamics II. Recuperado de: abata.sdsmt.edu

Ayres, R. U.; Ayres, L. W.; Martinas, K. (1996). Eco-Termodynamics: Exergy and Life-Cycle Analysis. INSEAD Working Paper 96/04/ EPS. Fontainebleau (France): INSEAD. 
Recuperado de: http://www.insead.edu/facultyresearch/research/doc.cfm?did=46686

Connelly, L.; Koshland, C. P. (2001). Exergy and industrial ecology - Part 1: An exergy-based definition of consumption and a thermodynamic interpretation of ecosystem evolution. Exergy, 1(3), 146-165. Recuperado de: http://www.sciencedirect.com/science/article/pii/S1164023501000218

Dincer, I.; Cengel, Y. A. (2001). Energy, Entropy and Exergy Concepts and Their Roles in Thermal Engineering. Entropy, 3, 116-149. Recuperado de: http://www.mdpi.com/10994300/3/3/116

Faria, S. H. B.; Zemp, R. J. (2005). Using exergy loss profiles and enthalpy-temperature profiles for the evaluation of thermodynamic efficiency in distillation columns. Thermal Eng., 4(1), 76-82. Recuperado de: http:// ojs.c3sl.ufpr.br/ojs/index.php/reterm/article/ view/3553

Gibbs, J.W. (1873). A Method of Geometrical Representation of the Thermodynamic Properties of Substances by Means of Surfaces. Transactions of the Connecticut Academy of Arts and Sciences, 2 Dec. 1873, p. 400.

Glasstone, S. (1946). Textbook of Physical Chemistry (2nd ed.). New York: Van Nostrand Co.

Green, D. W.; Perry, R. H. (2008). Perry's Chemical Engineers' Handbook (8th ed.). New York: McGraw Hill.

Kaiser, V.; Gourtia, J. P. (1985). The Ideal-Column Concept: Applying Exergy to Distillation. Chem. Eng. 92 (8), 45-53.

Linnhoff, B. (1979). Thermodynamic Analysis in the Design of Process Networks. (Ph. D. Dissertation). Leeds (UK): Univ. of Leeds, Dept. of Chem. Eng. Recuperado de: http://etheses. whiterose.ac.uk/2778/1/Linhoff_B_ChemicalEngineering _ PhD_1979.pdf

LowEx. (2002). Low Temperature Heating Systems: Increased Energy Efficiency and Improved Comfort. Brochure from the International Low Temperature Heating Systems Programme, IEA ECBCS. Recuperado de: lowex.net

Martínez, I. (1992). Termodinámica básica y aplicada. Capítulo 3 "Exergía”, pp. 45-49. Madrid (España): Dossat.
Meggers, F.;Ritter, V.; Goffin, P.; Baetschmann, M.; Leibundgut, H. (2012). Low exergy building systems implementation. Energy, 41(1), pp.48-55. Recuperado de: http:// www.sciencedirect.com/science/article/pii/ S0360544211004798

Morosuk, T.; Tsatsaronis, G. (2009). Advanced Exergy Analysis for Chemically Reacting Systems - Application to a Simple Open GasTurbine System. Int. J. of Thermodynamics, 12(3), 105-111. Recuperado de: http:// www.ijoticat.com/index.php/IJoT/article/ viewFile/245/227

Pisa, J; Agüero, C, Andina, R. (2010). Límites en el uso de balances de exergía en los sistemas de cogeneración. Revista CET, 32, 32-36. Recuperado de: http://www.herrera.unt.edu. ar/revistacet/?page_id $=310$

Pons, M. (2009). On the Reference State when Ambient Temperature Fluctuates. Int J. of Thermodynamics, 12(3), 113-121. Recuperado de:http://www.ijoticat.com/index.php/ IJoT/article/viewFile/246/228

Rosen, M. A.; Dincer, I. (2004). Effect of varying dead-state properties on energy and exergy analyses of thermal systems. Int.J.ofThermal Sciences, 43(2), 121-133. Recuperado de: http://www.sciencedirect.com/science/ article/pii/ S1290072903001418

Sciubba, E.; Wall, G. (2007). A Brief Commented History of Exergy From the Beginnings to 2004. Int. J. of Thermodynamics, 10(1), 1-26. Recuperado de: http:/www.ehakem.com/ index.php/IJoT/article/viewFile/184/170

Simpson, A. P.; Edwards, C. F. (2011). An exergybased framework for evaluating environmental impact. Energy, 36 (1), 1442-1459. Recuperado de:http://www.sciencedirect.com/ science/article/pii/S0360544211000260

Smith, J. M.; Van Ness, H. C.; Abbott, M. M. (2001). An Introduction to Chemical Engineering Thermodynamics (6th ed.). New York: McGraw Hill.

Smith, J. M.; Van Ness, H. C.; Abbott, M. M. (2004). An Introduction to Chemical Engineering Thermodynamics (7th ed.). New York: McGraw Hill.

Stremke, S.; van del Dobbelsteen, A.; Koh, J. (2011). Exergy landscapes: exploration of second-law thinking towards sustainable 
landscape design. Int. J. Exergy, Vol. 8, No. 2,148-174. Recuperado de: http://www.exergieplanning.nl/publicaties/2011\%20-\%20 Exergy\%20Landscapes\%20Stremke\%20Dobbelsteen $\% 20$ Koh.pdf

Shukuya, M. (2005). Towards Sustainable Arquitecture. Proceedings of the 2005 World Sustainable Building Conference, Tokio (Japan). Recuperado de: lowex.net

Szargut, J.; Morris, D. R.; Steward, F. R. (1988). Exergy Analysis of Thermal, Chemical and Metalurgical Processes. New York: Hemisphere Publishing Corp.

Tsatsaronis, G. (1999). Strengths and Limitations of Exergy Analysis. In: Bejan, A. \& Mamut, E. Thermodynamic Optimization of Complex Energy Systems, pp. 93-100. Dordrecht: Kluwer Academic Publishers. Recuperado de: http://www.researchgate.net/ publication/235928944_1999-(FP)Strengths_and_limitations_of_exergy_analysis/file/ 9fcfd5145a781a9468.pdf

Tsatsaronis, G. (2007). Comments on the Paper 'A Brief Commented History of Exergy from the Beginnings to 2004'. Int. J. of Thermodynamics, 10(4), 187-192. Recuperado de: http://www.ijoticat.com/index.php/ IJoT/article/viewFile/202/188

Van Wylen, G. J.; Sonntag, R. E. (1965). Fundamentals of Classical Thermodynamics (1st ed.).New York: Wiley.

Van Wylen, G. J.; Sonntag, R. E. (1976). Fundamentals of Classical Thermodynamics (2nd ed.). New York: Wiley.

Wall, G. (1977). Exergy - A Useful Concept. (Doctoral thesis, Chalmers Institute of
Technology) Göteborg, Sweden. Recuperado de: exergy.se

Wall, G. (1986). Exergy Flow in Industrial Processes. Recuperado de: exergy.se

Wall, G. (1991). On the Optimization of Refrigeration Machinery. Int. J. of Refrigeration, 14, 336-340. Recuperado de: exergy.se

Wall, G. (1992). A Bibliography of Exergy. Recuperado de: exergy.se

Zhinhong, L.; Ben, H. (2000). Optimal Design of Heat of Heat Exchanger Network Synthesis based on Exergy-Economic Object and Strategy of Decomposition and Coordination. Journal of Chem. Ind. And Eng. (China), 51(1), 96-101. Recuperado de: http://www.hgxb.com.cn/EN/abstract/ abstract8681.shtml

Zvolinsky, A. (2006). On exergy analysis and entropy production minimisation in industrial ecology. (Doctoral thesis). Trondheim: Norwegian University of Science and Technology. Recuperado de: http://ntnu.diva-portal.org/smash/record. jsf? pid=diva2: 122541

\section{SOBRE EL AUTOR}

\section{René Alejandro Mora Casal}

Doctor en Ingeniería Química de la Atlantic International University de los EE.UU. Maister en Administración de Negocios con énfasis en Gerencia Industrial de la Universidad Interamericana de Costa Rica. Licenciado en Ingeniería Química de la Universidad de Costa Rica. Correo electrónico:morarene1@gmail.com 